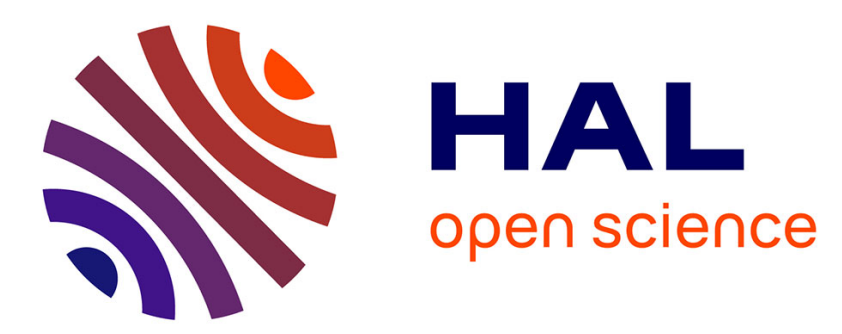

\title{
Uniformly convergent sliding mode-based observation for switched linear systems
}

Diego Mincarelli, Alessandro Pisano, Thierry Floquet, Elio Usai

\section{To cite this version:}

Diego Mincarelli, Alessandro Pisano, Thierry Floquet, Elio Usai. Uniformly convergent sliding modebased observation for switched linear systems. International Journal of Robust and Nonlinear Control, 2016, 26 (7), pp.1549-1564. 10.1002/rnc.3366 . hal-01709246

\section{HAL Id: hal-01709246 \\ https://hal.science/hal-01709246}

Submitted on 30 Mar 2018

HAL is a multi-disciplinary open access archive for the deposit and dissemination of scientific research documents, whether they are published or not. The documents may come from teaching and research institutions in France or abroad, or from public or private research centers.
L'archive ouverte pluridisciplinaire HAL, est destinée au dépôt et à la diffusion de documents scientifiques de niveau recherche, publiés ou non, émanant des établissements d'enseignement et de recherche français ou étrangers, des laboratoires publics ou privés. 


\title{
Uniformly Convergent Sliding Mode-based Observation for Switched Linear Systems
}

\author{
D. Mincarelli ${ }^{\mathrm{a}}$, A. Pisano ${ }^{\mathrm{b}}$, T. Floquet $^{\mathrm{c}}$ and E. Usai ${ }^{\mathrm{b}}$ \\ ${ }^{a}$ INRIA Lille - Nord Europe, France. \\ ${ }^{\mathrm{b}}$ Dept. of Electrical and Electronic Engineering (DIEE), Univ. of Cagliari, \\ Cagliari, Italy. \\ ${ }^{\mathrm{c}}$ LAGIS École Centrale de Lille, France.
}

\begin{abstract}
The problem of designing an observer capable of reconstructing the continuous and discrete state for a class of switched linear systems is addressed. A stack of dynamical observers, based on the Super-Twisting Algorithm (STA) with uniform convergence (i.e., finite time converging and with a maximal transient duration independent of the initial conditions) is considered, which provides an estimate of the continuous state and, at the same time, produces residual signals suitable for reconstructing the discrete state. An appropriate post-processing of the residuals is also suggested, which allows to speed up the reconstruction of the discrete state. Formal "distinguishability" conditions guaranteeing that the discrete state can be uniquely reconstructed are given. Lyapunov based proofs of convergence, and numerical simulations, support the proposed approach.
\end{abstract}

Key words: Switched systems; observer design ; second order sliding modes.

\section{INTRODUCTION}

In the last decade, the control community has devoted a great deal of attention to the study of hybrid/switched systems $[15,18,20]$. They represent a powerful tool to describe systems that exhibit switchings between several subsystems, inherently by nature or as a result of external control actions such as in switching supervisory control [23]. Switched systems and switched multi-controller

Email addresses: diego.mincarelli@inria.fr(D. Mincarelli), pisano@diee.unica.it (A. Pisano), thierry.floquet@ec-lille.fr (T.

Floquet), eusai@diee.unica.it (E. Usai). 
synthesis have numerous applications in the control of mechanical systems [24,8], automotive industry [4], switching power converters [13], aircraft and traffic control $[19,17]$, biological systems $[32,9]$, and many other fields [7]. Remarkable theoretical results involving switched systems have been achieved concerning their stability and stabilizability [22,21], controllability and reachability [28,29] and observability [31,33,2,28].

The problem of observer design for linear switched systems has been thoroughly investigated by the control community and different approaches have been proposed. The assumptions about the knowledge of the discrete state play a crucial role.

In the case of complete knowledge of the discrete state a Luenberger-like switched observer matching the currently active dynamics can be used and the problem is that of guaranteeing the stability of the switched error dynamics. In [1] it is shown that the observer gain matrices can be selected by solving a set of linear matrix inequalities. In [5], the approach is generalized to cover linear switched systems with unknown exogenous inputs. In [30], by adopting the notion of observability over an interval, borrowed from [33], an observer is designed for switched systems whose subsystems are not even required to be separately observable.

However, in some situations the active mode is unknown and needs to be estimated, along with the continuous state, by relying only on the continuous output measurements. Usually, in such case, the observer consists of two parts: a discrete state (or location) observer, estimating the active mode of operation, and a continuous observer that estimates the continuous state of the switched system.

In [3], the architecture of a hybrid observer consisting of both a discrete and continuous state identification part is presented, assuming partial knowledge of the discrete state, i.e. dealing with the case in which some discrete events causing the switchings are supposed to be measurable. When such a discrete output is not sufficient to identify the mode location, the information available from the continuous evolution of the plant is used to estimate the current mode. However, the "distinguishability" of the different modes, i.e. the property concerning the possibility to reconstruct univocally the discrete state, was not analysed.

The present work intrinsically differs from [3] in that we consider the case of completely unknown discrete state. In such a case the possibility to obtain an estimate of the current mode in a finite time is clearly important, not to say crucial. This is clear for instance from [26], where the authors focus on the continuous-time estimation problem and show that a bound to the estimation error can be given if the discrete mode is estimated correctly within a certain time. Additionally, for those switched systems admitting a dwell time, a guaranteed convergence of the discrete mode estimation taking place "sufficiently faster" that the dwell time is needed.

In view of these considerations sliding mode-based observers seem to be a suitable tool due to the attractive feature of finite-time convergence charac- 
terizing the sliding motions $[11,16,25,27]$. As a matter of fact, sliding mode observers have been successfully implemented to deal with the problem of state reconstruction for switched systems. In [6], an observer is proposed ensuring the reconstruction of the continuous and discrete state in finite time. In [12], the authors present an observer, based on the high-order sliding mode approach, for nonlinear autonomous switched systems. However in the above works, though guaranteeing the finite-time convergence, the convergence time depends on the initial conditions mismatch, and, as a consequence, the estimation convergence in a certain pre-specified time can be guaranteed only upon the existence of an a-priori known admissible domain for the system initial conditions.

\subsection{Main contribution and structure of the paper}

In the present paper we propose a stack of observers whose output injection is computed by relying on the modified Super-Twisting Algorithm, introduced in [10], that guarantees the so-called "uniform convergence" property, i.e. convergence is attained in finite-time and an upper bound to the transient time can be computed which does not depend on the initial conditions. We also show that, under some conditions, the discrete mode can be correctly reconstructed in finite-time after any switch independently of the observation error at the switching times. Using the continuous output of the switched system, the observer estimates the continuous state and, at the same time, produces suitable residual signals allowing the estimation of the current mode. We propose a residual "projection" methodology by means of which the discrete state can be reconstructed after a switching instant with a finite and pre-specified estimation transient time, allowing a more quick and reliable reconstruction of the discrete state. Additionally, we give structural "distinguishability" conditions in order to guarantee the possibility to reconstruct the discrete state univocally by processing the above mentioned residuals.

The paper structure is as follows. Section 2 formulates the problems under analysis and outlines the structure of the proposed scheme. Section 3 illustrates the design of the continuus state observers' stack by providing the underlying Lyapunov based convergence analysis. Section 4 deal with the discrete state estimation problem. Two approaches are proposed, one using the " asymptotically vanishing residuals" (Subsection 4.1) and another one, taking advantage of the above mentioned residuals' "projection" methodology ("uniform-time zeroed residuals", Subsection 4.2). Section 4.3 outlines the structural conditions addressing the "distinguishability" issues. Section 5 summarizes the proposed scheme and main results of this paper. Section 6 illustrates some simulation results and Section 7 gives some concluding remarks. 


\subsection{Notation}

For a vector $v=\left[v_{1}, v_{2}, \ldots, v_{n}\right]^{T} \in \mathbb{R}^{n}$ denote

$$
\operatorname{sign}(v)=\left[\operatorname{sign}\left(v_{1}\right), \operatorname{sign}\left(v_{2}\right), \ldots, \operatorname{sign}\left(v_{n}\right)\right]^{T} .
$$

Given a set $\mathcal{D} \subseteq \mathbb{R}^{n}$, let $\bar{v}_{j}(\mathcal{D})$ be the maximum value that can assume the $j$-th element of $v$ on $\mathcal{D}$. Denote as $\|M\|$ the 2-norm of a matrix $M$. For a square matrix $M$, denote as $\sigma(M)$ the spectrum of $M$, i.e. the set of all eigenvalues of $M$. Finally, denote as $\mathcal{N}^{\leftarrow}(M)$ the left null space of a matrix $M$.

\section{PROBLEM STATEMENT}

Consider the linear autonomous switched dynamics

$$
\begin{aligned}
\dot{x}(t) & =A_{\sigma} x(t) \\
y(t) & =C_{\sigma} x(t)
\end{aligned}
$$

where $x(t) \in \mathbb{R}^{n}$ represents the state vector, and $y(t) \in \mathbb{R}^{p}$ represents the output vector. The so-called switching law or discrete state $\sigma(t):[0, \infty) \rightarrow$ $\{1,2, \ldots, q\}$ determines the actual system dynamics among the possible $q$ "operating modes" which are represented, for system (2), by the set of matrix pairs $\left\{A_{1}, C_{1}\right\},\left\{A_{2}, C_{2}\right\}, \ldots\left\{A_{q}, C_{q}\right\}$. Without loss of generality, it is assumed that the output matrices $C_{i}, \forall i=1,2, \ldots, q$ are full row rank matrices. The switching law is a piecewise constant function with discontinuities at the switching time instants:

$$
\sigma(t)=\sigma_{k}, \quad t_{k} \leq t<t_{k+1}, \quad k=0,1, \ldots, \infty
$$

where $t_{0}=0$ and $t_{k}(k=1,2, \ldots)$ are the time instants at which the switches take place.

Definition 1 The dwell time is a constant $\Delta>0$ such that the switching times fulfill the inequality $t_{k+1}-t_{k} \geq \Delta$ for all $k=0,1, \ldots$.

The objective is to design an observer able to simultaneously estimate the discrete state $\sigma(t)$ and the continuous state $x(t)$ of system (2), by relying on the availability for measurements of the output vector $y(t)$.

We propose a design methodology based on a stack of sliding mode observers producing estimates of the evolution of the continuous state of the switched system. At the same time, suitable residuals are provided for identifying the actual value of the discrete state and, consequently, the specific observer of 
the stack that is producing an asymptotically converging estimate of the continuous state.

The observer structure, depicted in Fig. 1, mainly consists of two parts: a location observer and a continuous state observer. The location observer is devoted to the identification of the discrete state, i.e. the active mode of operation of the switched system, on the basis of some residuals signals produced by the continuous observer.

The continuous state observer receives as input the output vector $y(t)$ of the switching system and, using the location information provided by the location observer, produces an estimation of the continuous state of the system.

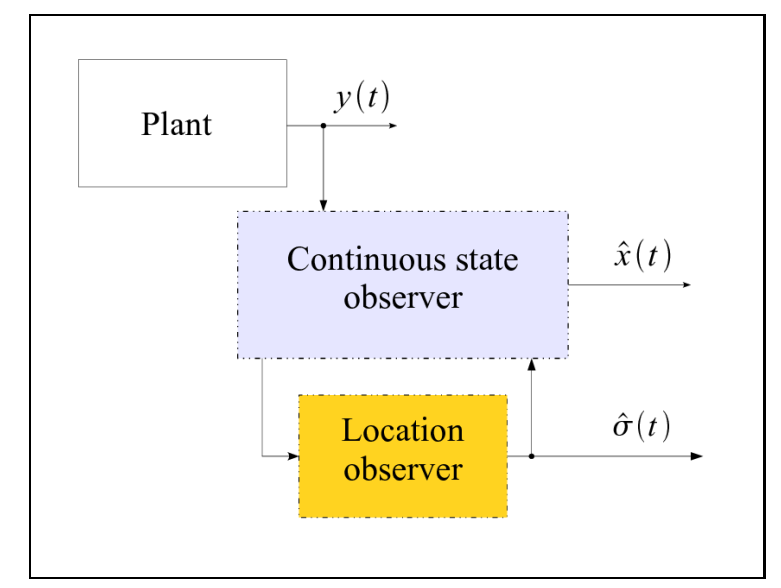

Fig. 1. Observer structure

We assume that the switching time instants fulfill the next

Assumption 1 System (2) admits a minimal dwell time $\Delta>0$, where $\Delta$ is a known constant.

Moreover, it is assumed that:

Assumption 2 The state trajectories $x(t)$ of systems (2)-(3) evolve into an a priori known, arbitrarily large, compact domain $\mathcal{D} \subset \mathbb{R}^{n}$.

Finally, the proposed approach requires each subsystem to be observable:

Assumption 3 The pairs $\left(A_{i}, C_{i}\right)$ are observable $\forall i=1,2, \ldots, q$.

\section{Continuous state observer design}

Let us preliminarily consider, as suggested in [14], a family of nonsingular coordinate transformations such that the output vector $y(t)$ is a part of the 
transformed state $z(t)$, i.e.

$$
z(t)=\left[\begin{array}{l}
\xi(t) \\
y(t)
\end{array}\right]=T_{\sigma} x(t)
$$

where $\xi(t) \in \mathbb{R}^{(n-p)}$ and the transformation matrix is given by

$$
T_{\sigma}=\left[\begin{array}{c}
\left(N_{\sigma}\right)^{T} \\
C_{\sigma}
\end{array}\right]
$$

where the columns of $N_{i} \in \mathbb{R}^{n \times(n-p)}$ span the null space of $C_{i}, i=1,2, \ldots, q$. By Assumption 2, the trajectories $z(t)$ will evolve into some known compact domain $\mathcal{D}_{z}$.

The transformation (4) is always nonsingular, and the switched system dynamics (2) in the transformed coordinates are:

$$
\dot{z}(t)=\bar{A}_{\sigma} z(t)
$$

where

$$
\bar{A}_{\sigma}=T_{\sigma} A_{\sigma}\left(T_{\sigma}\right)^{-1}=\left[\begin{array}{cc}
\bar{A}_{\sigma 11} & \bar{A}_{\sigma 12} \\
\bar{A}_{\sigma 21} & \bar{A}_{\sigma 22}
\end{array}\right]
$$

A stack of $q$ dynamical observers, each one associated to a different mode of the switched system, is suggested as follows:

$$
\begin{array}{rlrl}
\dot{\hat{z}}_{i}(t) & =\bar{A}_{i} \hat{z}_{i}(t)+\bar{L}_{i} \nu_{i}(t), \quad \text { if } \hat{z}_{i} \in \mathcal{D}_{z} & i=1,2, \ldots, q, \\
\hat{z}_{i j}(t) & =\bar{z}_{j}\left(\mathcal{D}_{z}\right), \quad & \text { if } \hat{z}_{i j} \geq \bar{z}_{j}\left(\mathcal{D}_{z}\right) & j=1,2, \ldots, n
\end{array}
$$

where $\hat{z}_{i}=\left[\begin{array}{ll}\hat{\xi}_{i}, & \hat{y}_{i}\end{array}\right]^{T}$ is the state estimate provided by the $i$-th observer, $\nu_{i} \in \mathbb{R}^{p}$ represents the $i$-th observer injection input, yet to be designed, and $\bar{L}_{i} \in \mathbb{R}^{n \times p}$ takes the form

$$
\bar{L}_{i}=\left[\begin{array}{c}
L_{i} \\
-I
\end{array}\right]
$$

where $L_{i} \in \mathbb{R}^{(n-p) \times p}$ are observer gain matrices to be designed and $I$ is the identity matrix of dimension $p$. In the second of (8), which corresponds to a saturating projection of the estimated state, according to the a-priori known domain of evolution $\mathcal{D}_{z}$ of vector $z(t)$, the notation $\hat{z}_{i j}$ denotes the $j$-th element of the vector $\hat{z}_{i}$. 
By introducing the error variables

$$
e_{\xi}^{i}(t)=\hat{\xi}_{i}(t)-\xi(t), \quad e_{y}^{i}(t)=\hat{y}_{i}(t)-y(t), \quad i=1,2, \ldots, q,
$$

in view of (6) and of the first equation of (8) the next error dynamics can be easily obtained:

$$
\begin{aligned}
& \dot{e}_{\xi}^{i}(t)=\bar{A}_{i 11} e_{\xi}^{i}(t)+\bar{A}_{i 12} e_{y}^{i}(t)+\left(\bar{A}_{i 11}-\bar{A}_{\sigma 11}\right) \xi(t)+\left(\bar{A}_{i 12}-\bar{A}_{\sigma 12}\right) y(t)+L_{i} \nu_{i}(t) \\
& \dot{e}_{y}^{i}(t)=\bar{A}_{i 21} e_{\xi}^{i}(t)+\bar{A}_{i 22} e_{y}^{i}(t)+\left(\bar{A}_{i 21}-\bar{A}_{\sigma 21}\right) \xi(t)+\left(\bar{A}_{i 22}-\bar{A}_{\sigma 22}\right) y(t)-\nu_{i}(t)
\end{aligned}
$$

Then, by defining

$$
\varphi_{i \sigma}\left(e_{\xi}^{i}, e_{y}^{i}, \xi, y\right)=\bar{A}_{i 21} e_{\xi}^{i}(t)+\bar{A}_{i 22} e_{y}^{i}(t)+\left(\bar{A}_{i 21}-\bar{A}_{\sigma 21}\right) \xi(t)+\left(\bar{A}_{i 22}-\bar{A}_{\sigma 22}\right) y(t)
$$

one can rewrite (12) as

$$
\dot{e}_{y}^{i}(t)=\varphi_{i \sigma}\left(e_{\xi}^{i}, e_{y}^{i}, \xi, y\right)-\nu_{i}(t)
$$

Let us consider now the second equation of (8). We know that the trajectories $z(t)$ evolve inside a compact domain $\mathcal{D}_{z}$ and the "reset" relation $\hat{z}_{i j}(t)=$ $\bar{z}_{j}\left(\mathcal{D}_{z}\right)$, applied when the corresponding estimate leaves the domain $\mathcal{D}_{z}$, forces them to remain in the set $\mathcal{D}_{z}$. Such a "saturation" mechanism in the observer guarantees that the estimation errors $e_{\xi}^{i}$ and $e_{y}^{i}$ are always bounded. Consequently the functions $\varphi_{i j}$ are smooth enough, that is there exist a constant $\Phi$ such that the following inequality is satisfied

$$
\left\|\frac{d}{d t} \varphi_{i j}\left(e_{\xi}^{i}, e_{y}^{i}, \xi, y\right)\right\| \leq \Phi, \quad \forall i, j=1,2, \ldots, q
$$

Following [10], the observer injection term $\nu_{i}$ is going to be specified within the next Theorem which establishes some properties of the proposed observer stack that will be instrumental in our next developments.

Theorem 1 Consider the linear switched system (2), satisfying the Assumptions 1-3 along with the stack of observers (8), and the observer injection terms set according to

$$
\begin{aligned}
\nu_{i} & =k_{1} \phi_{1}\left(e_{y}^{i}\right)-\nu_{2 i} \\
\dot{\nu}_{2 i} & =-k_{2} \phi_{2}\left(e_{y}^{i}\right)
\end{aligned}
$$

where 


$$
\begin{aligned}
& \phi_{1}\left(e_{y}^{i}\right)=\left|e_{y}^{i}\right|^{1 / 2} \operatorname{sign}\left(e_{y}^{i}\right)+\mu\left|e_{y}^{i}\right|^{3 / 2} \operatorname{sign}\left(e_{y}^{i}\right) \\
& \phi_{2}\left(e_{y}^{i}\right)=\frac{1}{2} \operatorname{sign}\left(e_{y}^{i}\right)+2 \mu e_{y}^{i}+\frac{3}{2} \mu^{2}\left|e_{y}^{i}\right|^{2} \operatorname{sign}\left(e_{y}^{i}\right)
\end{aligned}
$$

with $\mu>0$ and the tuning coefficients $k_{1}$ and $k_{2}$ selected in the set:

$$
\begin{aligned}
& \mathcal{K}=\left\{\left(k_{1}, k_{2}\right) \in \mathbb{R}^{2} \mid 0<k_{1}<2 \sqrt{\Phi}, k_{2}>\frac{k_{1}^{2}}{4}+\frac{4 \Phi^{2}}{k_{1}^{2}}\right\} \\
& \cup\left\{\left(k_{1}, k_{2}\right) \in \mathbb{R}^{2} \mid k_{1}<2 \sqrt{\Phi}, k_{2}>2 \Phi\right\}
\end{aligned}
$$

Let $L_{i}$ be chosen such that

$$
\operatorname{Re}\left\{\sigma\left\{\bar{A}_{i 11}+L_{i} \bar{A}_{i 21}\right\}\right\} \leq-\gamma, \quad \gamma>0
$$

Then, for sufficiently large $\Phi$ and $\gamma$, there exists an arbitrarily small time $T^{*}<<\Delta$ independent of $e_{y}^{i}\left(t_{k}\right)$ such that, for all $k=0,1, \ldots, \infty$ and for some $\alpha>0$, the next properties hold along the time intervals $t \in\left[t_{k}+T^{*}, t_{k+1}\right)$ :

$$
\begin{gathered}
e_{y}^{i}(t)=0 \quad \forall i \\
\nu_{i}(t)=\varphi_{i \sigma}\left(e_{\xi}^{i}, 0, \xi, y\right) \quad \forall i \\
\left\|e_{\xi}^{\sigma_{k}}(t)\right\| \leq \alpha e^{-\gamma\left(t-t_{k}-T^{*}\right)}
\end{gathered}
$$

Proof. The theorem can be proven by showing the uniform (i.e. independent of the initial condition) time convergence of $e_{y}^{i}$ to zero for all the $q$ observers, after each switching, and analyzing the dynamics of the error variables $e_{\xi}^{i}$ once the trajectories are restricted on the surfaces

$$
\mathcal{S}_{o}^{i}=\left\{\left(e_{\xi}^{i}, e_{y}^{i}\right): e_{y}^{i}=0\right\}
$$

Considering the input injection term (16) into (14) the output error dynamics are given by

$$
\dot{e}_{y}^{i}=\varphi_{i \sigma}-k_{1} \phi_{1}\left(e_{y}^{i}\right)+\nu_{2 i}
$$

By introducing the new coordinates

$$
\psi_{i}=\nu_{2 i}+\varphi_{i \sigma}
$$

and considering (17) one obtains the system

$$
\begin{aligned}
\dot{e}_{y}^{i} & =-k_{1} \phi_{1}\left(e_{y}^{i}\right)+\psi_{i} \\
\dot{\psi}_{i} & =-k_{2} \phi_{2}\left(e_{y}^{i}\right)+\frac{d}{d t} \varphi_{i \sigma}
\end{aligned}
$$


In light of (15), system (28)-(29) is formally equivalent to that dealt with in [10], where suitable Lyapunov analysis was used to prove the uniform-time convergence to zero of $e_{y}^{i}$ and $\psi_{i}$, i.e. $e_{y}^{i}=0$ and $\psi_{i}=0$ on the interval $t \in\left[t_{k}+T^{*}, t_{k+1}\right)$, where $T^{*}$ is an arbitrarily small transient time independent of $e_{y}^{i}\left(t_{k}\right)$. Consequently $\dot{e}_{y}^{i}=0$ and, from equation (14), condition (23) is satisfied too. By substituting (23) into (11) with $e_{y}^{i}=0$, it yields the next equivalent dynamics of the error variables:

$$
\dot{e}_{\xi}^{i}(t)=\bar{A}_{i 11} e_{\xi}^{i}(t)+\left(\bar{A}_{i 11}-\bar{A}_{\sigma 11}\right) \xi(t)+\left(\bar{A}_{i 12}-\bar{A}_{\sigma 12}\right) y(t)+L_{i} \varphi_{i \sigma}\left(e_{\xi}^{i}, 0, \xi, y\right)
$$

where

$$
\varphi_{i \sigma}\left(e_{\xi}^{i}, 0, \xi, y\right)=\bar{A}_{i 21} e_{\xi}^{i}(t)+\left(\bar{A}_{i 21}-\bar{A}_{\sigma 21}\right) \xi(t)+\left(\bar{A}_{i 22}-\bar{A}_{\sigma 22}\right) y(t)
$$

Finally, by defining the following matrices

$$
\begin{aligned}
\tilde{A}_{i} & =\left(\bar{A}_{i 11}+L_{i} \bar{A}_{i 21}\right) \\
\Delta A_{i \sigma}^{\xi} & =\left(\bar{A}_{i 11}-\bar{A}_{\sigma 11}\right)+L_{i}\left(\bar{A}_{i 21}-\bar{A}_{\sigma 21}\right) \\
\Delta A_{i \sigma}^{y} & =\left(\bar{A}_{i 12}-\bar{A}_{\sigma 12}\right)+L_{i}\left(\bar{A}_{i 22}-\bar{A}_{\sigma 22}\right)
\end{aligned}
$$

equation (30) can be rewritten as

$$
\dot{e}_{\xi}^{i}(t)=\tilde{A}_{i} e_{\xi}^{i}(t)+\Delta A_{i \sigma}^{\xi} \xi(t)+\Delta A_{i \sigma}^{y} y(t)
$$

It is worth noting that for the correct observer (i.e., that having the index $i=\sigma_{k}$ which matches the current mode of operation $\left.\sigma(t)\right)$ one has $\Delta A_{i \sigma}^{\xi}=$ $\Delta A_{i \sigma}^{y}=0$. Hence, along every time intervals $t \in\left[t_{k}+T^{*}, t_{k+1}\right)$, with $k=$ $0,1, \ldots, \infty$, the error dynamics of the correct observer are given by

$$
\dot{e}_{\xi}^{\sigma_{k}}(t)=\tilde{A}_{\sigma_{k}} e_{\xi}^{\sigma_{k}}(t)
$$

which is asymptotically stable by (21). Since by Assumption 3 the pairs $\left(A_{i}, C_{i}\right)$ are all observable, the pairs $\left(\bar{A}_{i 11}, \bar{A}_{i 21}\right)$ are also observable, which motivates the tuning condition (21). The solution of (34) fulfills the relation (24).

Remark 1 Equation (34) means that the estimation $\hat{\xi}_{i}$ provided by the "correct" observer, i.e. the observer associated to the mode $\sigma_{k}$ activated during the interval $t \in\left[t_{k}, t_{k+1}\right)$, at time $t_{k}+T^{*}$ starts to converge exponentially to the real continuous state $\xi$. By appropriate choice of $L_{i}$ the desired rate of convergence can be obtained.

Remark 2 It is worth stressing that the time $T^{*}$ is independent of the initial error at each time $t_{k}$ and can be made as small as desired (and in particular such that $T^{*}<<\Delta$ ) by tuning the parameters of the observers properly. 


\section{Discrete state estimation}

In the previous section it was shown that there is one observer in the stack that provides the asymptotic reconstruction of the continuous state of the switched system (2). However, the index of such "correct" observer is the currently active mode, which is still unknown to the designer, hence the scheme needs to be complemented by a discrete mode observer. In the next subsections we present two methods for reconstructing the discrete state of the system by suitable processing of the observers' output injections.

\subsection{Asymptotically vanishing residuals}

Along the time intervals $\left[t_{k}+T^{*}, t_{k+1}\right)$ the observers' output injection vectors (23) satisfy the following relationship:

$$
\nu_{i}(t)=\left\{\begin{array}{cc}
\bar{A}_{\sigma_{k} 21} e_{\xi}^{\sigma_{k}}(t) & \text { for } i=\sigma_{k} \\
\bar{A}_{i 21} e_{\xi}^{i}(t)+\left(\bar{A}_{i 21}^{i}-\bar{A}_{\sigma_{k} 21}\right) \xi(t)+\left(\bar{A}_{i 22}-\bar{A}_{\sigma_{k} 22}\right) y(t) & \text { for } i \neq \sigma_{k}
\end{array}\right.
$$

It turns out that along the time intervals $\left[t_{k}+T^{*}, t_{k+1}\right)$ the norm of the injection term of the correct observer will be asymptotically vanishing in accordance with

$$
\left\|\nu_{\sigma_{k}}(t)\right\| \leq A_{M 21} \alpha e^{-\gamma\left(t-t_{k}-T^{*}\right)} \rightarrow 0
$$

where

$$
A_{M 21}=\sup _{i \in\{1,2, \ldots, q\}}\left\|\bar{A}_{i 21}\right\|
$$

The asymptotic nature of the convergence to zero of the residual vector corresponding to the correct observer is due to the dynamics (34) of the error variable $e_{\xi}^{\sigma_{k}}(t)$, which in fact tends asymptotically to zero.

\subsection{Uniform-time zeroed residuals}

By making the injection signals insensitive to the dynamics of $e_{\xi}^{i}(t)$ it is possible to obtain a uniform-time zeroed residual for the correct observer, i.e. a residual which is exactly zeroed after a finite time $T^{*}$ following any switch, independently of the error at the switching time. Let us make the next assumption. 
Assumption 4 For all $i=1,2, \ldots, q$, the submatrices $\bar{A}_{i 21}$ are not full row rank.

The major consequence of Assumption 4 is that $\mathcal{N}^{\leftarrow}\left(\bar{A}_{i 21}\right)$ is not trivial. Let $U_{i}$ be a basis for $\mathcal{N}^{\leftarrow}\left(\bar{A}_{i 21}\right)$ (i.e. $U_{i} \bar{A}_{i 21}=0$ ) and denote

$$
\bar{\nu}_{i}(t)=U_{i} \nu_{i}(t)
$$

Clearly, by (35), on the interval $\left[t_{k}+T^{*}, t_{k+1}\right)$ one has that

$$
\bar{\nu}_{i}(t)=\left\{\begin{array}{cc}
0 & \text { for } i=\sigma_{k} \\
-U_{i} A_{\sigma_{k} 21} \xi(t)+U_{i}\left(\bar{A}_{i 22}-\bar{A}_{\sigma_{k} 22}\right) y(t) & \text { for } i \neq \sigma_{k}
\end{array}\right.
$$

It turns out that starting from the time $t_{k}+T^{*}$ the norm of the injection term of the correct observer will be exactly zero, i.e. $\left\|\bar{\nu}_{\sigma_{k}}(t)\right\|=0 \forall t \in\left[t_{k}+T^{*}, t_{k+1}\right)$.

In order to reconstruct univocally the discrete state, it must be guaranteed that the "wrong" residuals cannot stay identically at zero. In the following section we shall derive a structural condition on the system matrices guaranteeing that the uniform-time zeroed residuals associated to the "wrong" observers cannot stay at the origin.

\subsection{Uniqueness of the reconstructed switching law}

The main property allowing the discrete mode reconstruction is that, after a finite time $T^{*}$ following any switch, the residual corresponding to the "correct" observer converges to zero, according to (35), or it is exactly zero if the uniform-time zeroed residuals (38) are used. In order to reconstruct the discrete state univocally, all the other residuals (i.e. those having indexes corresponding to the non activated modes) must be separated from zero. In what follows the uniform-time zeroed residuals (38) are considered as they provide better performance and faster reconstruction capabilities. Nevertheless, analogue considerations can be made in the case of the asymptotically vanishing residuals (35).

Definition 2 Given the switched system (2), a residual $\bar{\nu}_{i}(t)$ is said to be non vanishing if $x(t) \neq 0$ almost everywhere implies $\bar{\nu}_{i}(t) \neq 0$ almost everywhere $\forall i \neq \sigma_{k}$, that is the residuals corresponding to the "wrong" observers cannot be identically zero on a time interval unless the state of the system is identically zero in the considered interval. 
Next Lemma establishes an "observability-like" requirement guaranteeing that that the uniform-time zeroed residuals (39) are non vanishing.

Lemma 1 The uniform-time zeroed residuals $\bar{\nu}_{i}(t)$ in (39) are non vanishing if and only if the pairs $\left(\bar{A}_{j}, \bar{C}_{i j}\right)$ are observable $\forall i \neq j$, where $\bar{A}_{j}(j=1,2, \ldots, q)$ are the state matrices of system (6) and $\bar{C}_{i j}=\left[-U_{i}^{T} \bar{A}_{j 21} U_{i}^{T}\left(\bar{A}_{i 22}-\bar{A}_{j 22}\right)\right]$.

Proof. Along the time interval $\left[t_{k}+T^{*}, t_{k+1}\right)$, the "wrong" residuals $\bar{\nu}_{i}(t)$ in (39), i.e. those with index $i \neq \sigma_{k}$, are related to the state $z(t)$ of system (6) as

$$
\bar{\nu}_{i}(t)=\bar{C}_{i \sigma_{k}} z(t)
$$

where $z(t)$ is the solution of

$$
\dot{z}(t)=\bar{A}_{\sigma_{k}} z(t)
$$

It is well known that if the pair $\left(\bar{A}_{\sigma_{k}}, \bar{C}_{i \sigma_{k}}\right)$ of system (40)-(41) is observable, then $\bar{\nu}_{i}(t)$ is identically zero if and only if $z(t)$ (and, thus, $\left.x(t)\right)$ is null. Therefore, to extend this property over all the intervals $t_{k}+T^{*} \leq t<t_{k+1}$ with $k=0,1, \ldots, \infty$, all the pairs $\left(\bar{A}_{j}, \bar{C}_{i j}\right) \forall i \neq j$ have to be observable.

Assumption 5 The state trajectories $x(t)$ in (2) are not zero almost everywhere and all the residuals $\bar{\nu}_{i}(t)$ in (38) are non vanishing according to Definition (2).

Thus if Assumption 5 is fulfilled, only the residual associated to the correct observer will be zero, while all the others will have a norm separated from zero. It is clear that if such a situation can be guaranteed, then the discrete mode can be univocally reconstructed by means of simple decision logic, as discussed in Theorem 2 of the next Section.

Remark 3 If Assumption 4 is not satisfied the residual (38) cannot be considered. Nevertheless, by considering the asymptotically vanishing residuals (35), similar structural conditions guaranteeing that the "wrong" residuals cannot be identically zero can be given. Consider the following extended vector:

$$
z_{e}^{i}(t)=\left[\begin{array}{c}
e_{\xi}^{i}(t) \\
\xi(t) \\
y(t)
\end{array}\right]
$$

From (6), (33) and (35) the following system can be considered on the interval $\left[t_{k}+T^{*}, t_{k+1}\right)$ : 


$$
\begin{aligned}
\dot{z}_{e}^{i}(t) & =\mathcal{A}_{i \sigma_{k}} z_{e}^{i}(t) \\
\nu_{i}(t) & =\mathcal{C}_{i \sigma_{k}} z_{e}^{i}(t)
\end{aligned}
$$

where

$$
\mathcal{A}_{i \sigma_{k}}=\left[\begin{array}{cc}
\tilde{A}_{i} \Delta A_{i \sigma_{k}}^{\xi} \Delta A_{i \sigma_{k}}^{y} \\
0 & \bar{A}_{\sigma_{k}}
\end{array}\right] \quad \mathcal{C}_{i \sigma_{k}}=\left[\begin{array}{c}
\bar{A}_{i 21} \\
\bar{A}_{i 21}-\bar{A}_{\sigma_{k} 21} \\
\bar{A}_{i 22}-\bar{A}_{\sigma_{k} 22}
\end{array}\right]^{T}
$$

The asymptotically vanishing residuals (35) are non vanishing if the pairs $\left(\mathcal{A}_{i j}, \mathcal{C}_{i j}\right)$ are observable $\forall i \neq j$.

\section{Continuous and discrete state observer}

The proposed methodology of continuous and discrete state estimation is summarized in the next

Theorem 2 Consider the linear switched system (2), fulfilling the Assumption 1-5, and the observer stack (8) described in the previous Theorem 1. Consider the next evaluation signal

$$
\bar{\rho}_{i}(t)=\int_{t-\epsilon}^{t}\left\|\bar{\nu}_{i}(\tau)\right\| d \tau
$$

where $\epsilon$, is a small time delay, and the next active mode identification logic:

$$
\hat{\sigma}(t)=\operatorname{argmin}_{i \in\{1,2, \ldots, q\}} \bar{\rho}_{i}(t)
$$

Then, the discrete state estimation will be such that

$$
\hat{\sigma}(t)=\sigma(t), \quad t_{k}+T^{*}+\epsilon \leq t \leq t_{k+1}, \quad k=0,1, \ldots, \infty
$$

and the continuous state estimation given by

$$
\hat{x}(t)=\left(T_{\hat{\sigma}}\right)^{-1}\left[\begin{array}{l}
\hat{\xi}_{\hat{\sigma}}(t) \\
\hat{y}_{\hat{\sigma}}(t)
\end{array}\right]
$$


will be such that

$$
\|\hat{x}(t)-x(t)\| \leq \alpha e^{-\gamma\left(t-t_{k}-T^{*}\right)} \quad \forall t \in\left[t_{k}+T^{*}, t_{k+1}\right)
$$

\section{Proof.}

By considering (39) which, specified for the correct observer $\left(i=\sigma_{k}\right)$, guarantees that

$$
\bar{\nu}_{\sigma_{k}}(t)=0, \quad t_{k}+T^{*} \leq t \leq t_{k+1}
$$

along with the Assumption 5 , whose main consequence is that $\bar{\nu}_{i}(t)$ cannot be identically zero over an interval when $i \neq \sigma(t)$, it follows that it is always possible to find a threshold $\eta$ such that for the evaluation signals $\bar{\rho}_{i}(t)$ in (45) one has

$$
\begin{aligned}
\bar{\rho}_{i}(t)>\eta, & t_{k}+T^{*}+\epsilon \leq t<t_{k+1}, \quad i \neq \sigma_{k} \\
\bar{\rho}_{\sigma_{k}}(t) \leq \eta, & t_{k}+T^{*}+\epsilon \leq t<t_{k+1} .
\end{aligned}
$$

Thus the mode decision logic (46) provides the reconstruction of the discrete state after the finite time $T^{*}+\epsilon$ successive to any switching time instant, i.e.

$$
\hat{\sigma}(t)=\sigma_{k}, \quad t_{k}+T^{*}+\epsilon \leq t<t_{k+1}
$$

The second part of the Theorem 2 concerning the continuous state estimation can be easily proven by considering the coordinate transformation (4) and Theorem 1, which imply (49).

Remark 4 We assumed that the state trajectories are not zero almost everywhere (Assumption 5). As a result the wrong residuals can occasionally cross the zero value. This fact motivates the evaluation signal introduced in (45): considering a window of observation for the residuals, all the wrong residuals will be separated from zero, while only the correct one can stay close to zero during a time interval of nonzero length.

The architecture of the observer is shown in Fig. 2.

Remark 5 It is possible to develop the same methodology if the residual (35) is considered instead of (38). The evaluation signal

$$
\rho_{i}(t)=\int_{t-\epsilon}^{t}\left\|\nu_{i}(\tau)\right\| d \tau .
$$




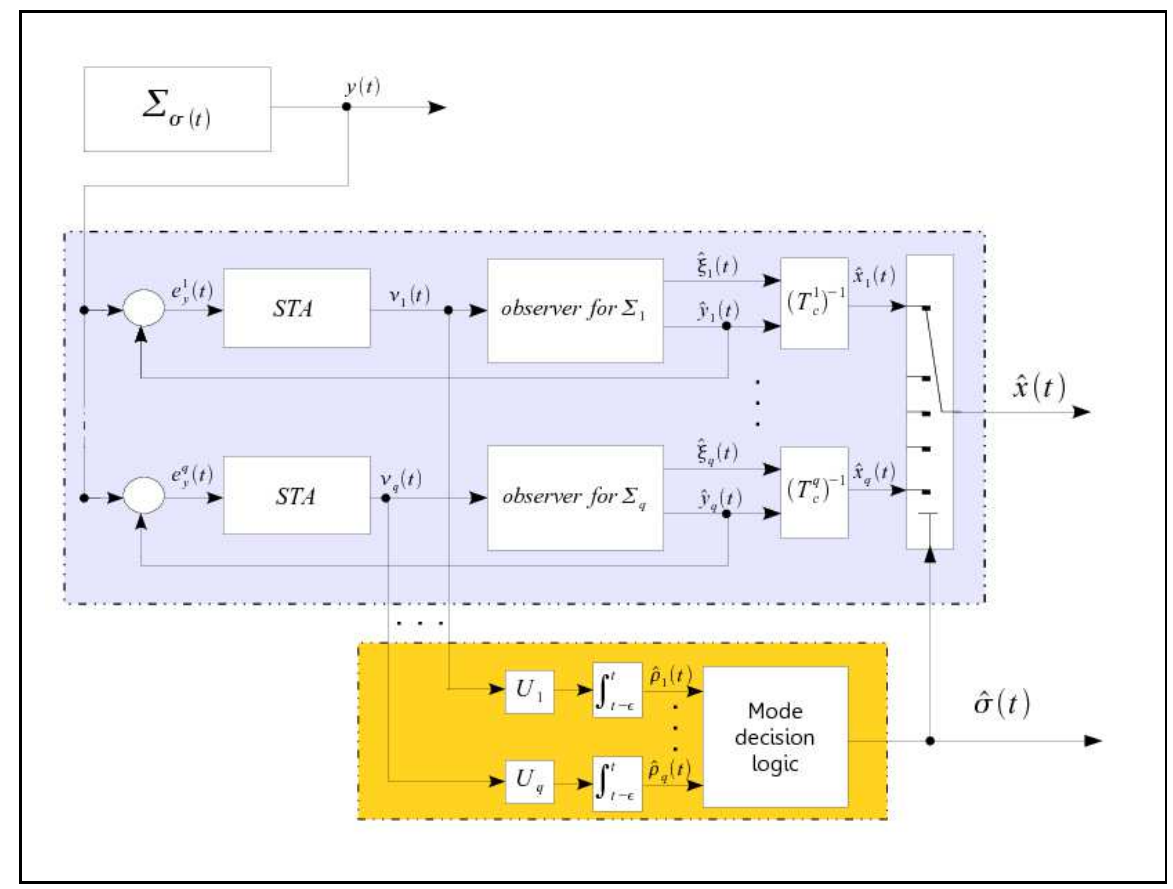

Fig. 2. Continuous and discrete state observer

can be used to identify the discrete state. However, the time to identify the discrete mode will be bigger than the one of Theorem 2 since the vanishing transient of the error variable $e_{\xi}^{i}$ is needed to last for a while, starting from the finite time $t_{k}+T^{*}$.

\section{SIMULATION RESULTS}

In this section, we discuss a simulation example to show the effectiveness of our method. Consider a switched linear system as in (2) with $q=3$ modes described by the matrices

$$
\begin{gathered}
A_{1}=\left[\begin{array}{rrr}
0.1 & 0.6 & -0.4 \\
-0.5 & -0.8 & 1 \\
0.1 & 0.4 & -0.7
\end{array}\right], A_{2}=\left[\begin{array}{rrr}
-0.2 & 0.3 & -0.8 \\
-0.2 & -0.4 & 0.8 \\
1 & 0.6 & -0.3
\end{array}\right], A_{3}=\left[\begin{array}{rrr}
-0.8 & -0.5 & 0.2 \\
-0.5 & -0.1 & -0.5 \\
-0.3-0.2 & 0.3
\end{array}\right] \\
C_{1}=\left[\begin{array}{lll}
1 & 0 & 0 \\
0 & 1 & 0
\end{array}\right], C_{2}=\left[\begin{array}{lll}
1 & 0 & 0 \\
0 & 0 & 1
\end{array}\right], C_{3}=\left[\begin{array}{lll}
0 & 0 & 1 \\
0 & 1 & 0
\end{array}\right]
\end{gathered}
$$

The system starts from the mode 1 with the initial conditions $x(0)=[-3,-1,6]^{T}$ 
and evolves switching between the three modes according to the switching law shown in Fig. 3. After the coordinate transformation (4) obtained by the transformation matrices

$$
T_{1}=\left[\begin{array}{lll}
0 & 0 & 1 \\
1 & 0 & 0 \\
0 & 1 & 0
\end{array}\right], T_{2}=\left[\begin{array}{rrr}
0 & -1 & 0 \\
1 & 0 & 0 \\
0 & 0 & 1
\end{array}\right], T_{3}=\left[\begin{array}{rrr}
-1 & 0 & 0 \\
0 & 0 & 1 \\
0 & 1 & 0
\end{array}\right]
$$

the system is in the proper form to apply our estimation procedure. Since the pairs $\left(A_{i}, C_{i}\right)$ are all observable for $i=1,2,3$, the stack of observers (8) can be implemented. By properly tuning the parameters of the STA-based observers according to (20), the components of the vector error $e_{y}$ are exactly zero for each observer after a time $T^{*}$ subsequent to any switch (Fig. 4) as proven in Theorem 1. On the contrary, the error $e_{\xi}$ at time $t_{k}+T^{*}$ starts to converge exponentially to zero only for the correct observer, as shown in Fig. 5. Notice that the three signals occasionally cross the zero, but only the one corresponding to the correct observer remains zero on a time interval. The gains of the observers $L_{i}$ are chosen such that the eigenvalues of the matrices $\tilde{A}_{i}$ in (32) governing the error dynamics (33) are located at -5 .

Since Assumption 5 is satisfied, the discrete mode can be univocally identified. To this end let us consider the asymptotically vanishing residuals (35) and the uniform-time zeroed residuals (39). The simulations confirm that both signals stay at zero only for the correct observer. Moreover, the signal corresponding to the asymptotically vanishing residual is in general "slower" than the signal corresponding to the uniform-time zeroed residual. In order to highlight the different behaviours of the two signals, we reported in Fig. 6 the dynamics of the two residuals provided by the third observer when the mode 3 becomes active at $t=20$. The evaluation signal obtained with the uniform-time zeroed residuals allows us a faster estimation of the switching law, as compared to their asymptotic counterparts. In Fig. 7 the actual and the reconstructed switching law are depicted by using the two different evaluation signals.

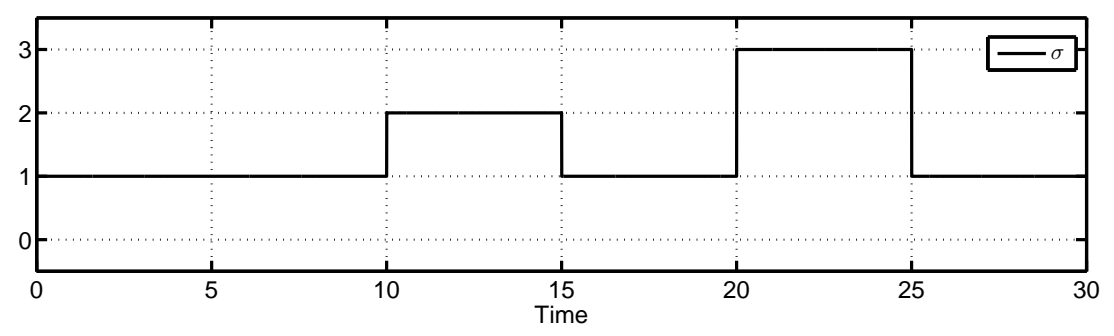

Fig. 3. Actual switching signal. 

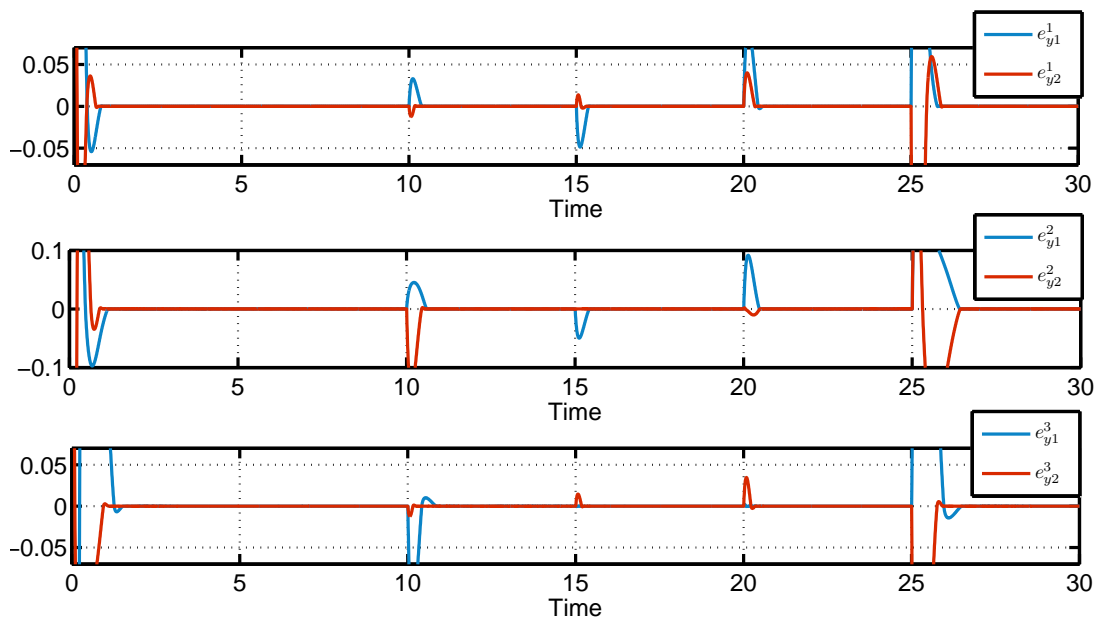

Fig. 4. Estimation vector error $e_{y}$ corresponding to the three observers.

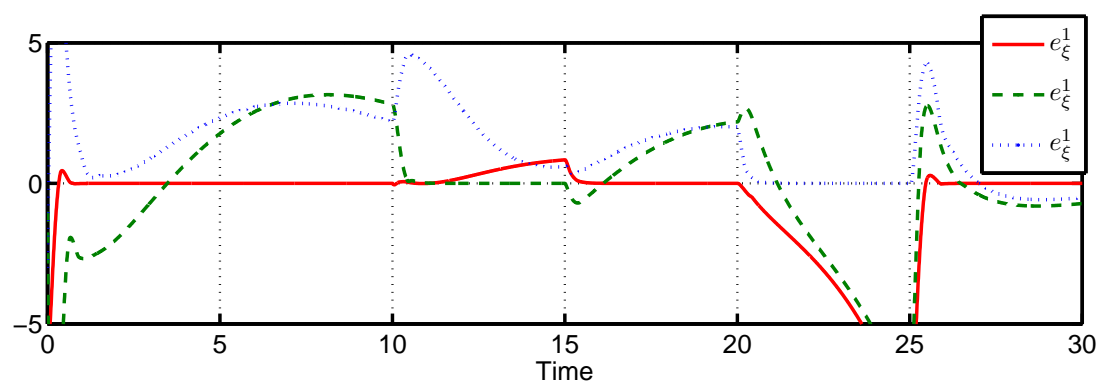

Fig. 5. Estimation error $e_{\xi}$ corresponding to the three observers.

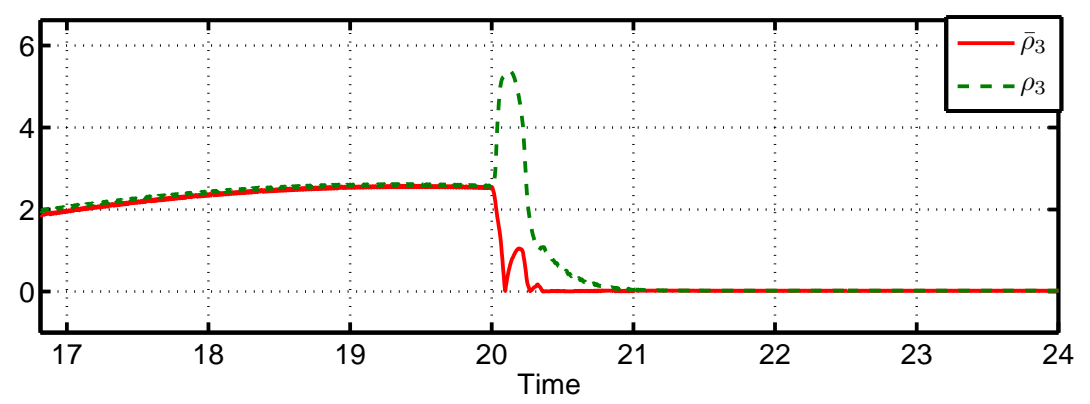

Fig. 6. Evaluation signal used to estimate the discrete state.

\section{CONCLUSIONS}

The problem of simultaneous continuous and discrete state reconstruction has been tackled for linear autonomous switched systems. The main ingredient of the proposed approach is an appropriate stack of high-order sliding mode observers used both as continuous state observers and as residual generators for discrete mode identification. As a novelty, a procedure has been devised to algebraically process the residuals in order to reconstruct the discrete state 


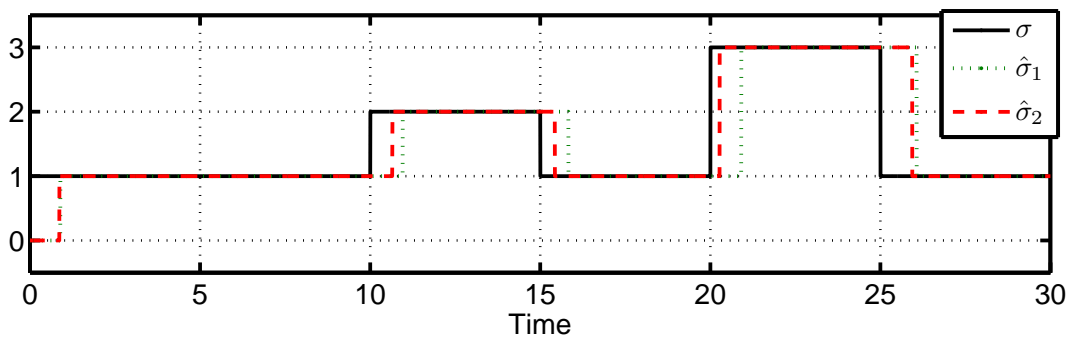

Fig. 7. Actual and reconstructed switching signals.

after a finite time that can be arbitrarily small, and, additionally, conditions ensuring the identifiability of the system modes are derived in terms of the original system matrices. An original "projection" procedure has been proposed, leading to the so-called uniform-time zeroed residuals, which allows a faster reconstruction of the active mode as compared to the (however feasible) case when such projection is no adopted. The same observer can be designed in the case of forced switched systems, too. However, further investigation is needed concerning the underlying conditions to univocally reconstruct the discrete state, which would be affected by the chosen input too.

\section{ACKOWLEDGMENTS}

A. Pisano gratefully acknowledges the financial support from the Ecole Centrale de Lille under the 2013 ECL visiting professor program.

\section{References}

[1] A. Alessandri and P. Coletta. Switching observers fo continuous-time and discrete-time linear systems. In Proceedings of the 2001 American Control Conference, pages 2516-2521, Arlington, Virginia, 2001.

[2] M. Babaali and G. J. Pappas. Observability of switched linear systems in continuous time. In Proceedings of the 8th international conference on Hybrid Systems: computation and control, HSCC'05, pages 103-117, Berlin, Heidelberg, 2005. Springer-Verlag.

[3] A. Balluchi, L. Benvenuti, M.D. Di Benedetto, and A.L. SangiovanniVincentelli. Design of observers for hybrid systems. In Hybrid Systems: Computation and Control, volume 2289 of LNCS, pages 76-89. Springer-Verlag, 2002.

[4] Andrea Balluchi, Maria Domenica Di Benedetto, Claudio Pinello, C. Rossi, and Alberto L. Sangiovanni-Vincentelli. Hybrid control in automotive applications: the cut-off control. Automatica, 35(3):519-535, 1999. 
[5] F. Bejarano and A. Pisano. Switched observers for switched linear systems with unknown inputs. IEEE Transactions on Automatic Control, 56(3):681$686,2011$.

[6] Francisco J. Bejarano and Leonid Fridman. State exact reconstruction for switched linear systems via a super-twisting algorithm. Intern. J. Syst. Sci., 42(5):717-724, 2011.

[7] M.S. Branicky. Studies in hybrid systems: Modeling, analysis, and control. PhD thesis, 1995.

[8] C. Chevallereau, E. R. Westervelt, and J. W. Grizzle. Asymptotically stable running for a five-link, four-actuator, planar bipedal robot. International Journal of Robotics Research, 24:431-464, 2005.

[9] E. Cinquemani, A. Milias, and John Lygeros. Identification of genetic regulatory networks: A stochastic hybrid approach. IFAC World Congress, 17, 2008.

[10] E. Cruz-Zabala, J.A. Moreno, and L.M. Fridman. Uniform robust exact differentiator. IEEE Transactions on Automatic Control, 56(11):2727-2733, June 2011.

[11] J. Davila, L. Fridman, and A. Levant. Second-order sliding-mode observer for mechanical systems. IEEE Trans. Automat. Contr., 50(11):1785-1789, 2005.

[12] J. Davila, A. Pisano, and E. Usai. Continuous and discrete state reconstruction for nonlinear switched systems via high-order sliding-mode observers. International Journal of Systems Science, 42(5):725-735, 2011.

[13] Willem L. De Koning. Brief digital optimal reduced-order control of pulsewidth-modulated switched linear systems. Automatica, 39(11):1997-2003, 2003.

[14] C. Edwards and S.K. Spurgeon. Sliding Mode Control: Theory and applications. Taylor and Francis, London, 1998.

[15] S. Engell. Modelling and analysis of hybrid systems. Mathematics and Computers in Simulation, 46:445-464, 1998.

[16] Thierry Floquet, Chris Edwards, and Sarah Spurgeon, K. On sliding mode observers for systems with unknown inputs. International Journal of Adaptive Control and Signal Processing, 21(8-9):638-656, 2007.

[17] William Glover and John Lygeros. A stochastic hybrid model for air traffic control simulation. HSCC, 2993:372-386, 2004.

[18] R. Goebel, R.G. Sanfelice, and A.R. Teel. Hybrid dynamical systems. IEEE Control Systems Magazine, 29:28-93, 2009.

[19] M. Kamgarpour, M. Soler, C.J. Tomlin, J. Olivares, and J. Lygeros. Hybrid Optimal Control for Aircraft Trajectory Design with a Variable Sequence of Modes. IFAC World Congress, 2011.

[20] D. Liberzon and A.S. Morse. Basic problems in stability and design of switched systems. IEEE Control Systems Magazine, 19:59-70, 1999. 
[21] Daniel Liberzon. Switching in Systems and Control. Systems and Control: Foundations and Applications. Birkhäuser, Boston, MA, 2003.

[22] H. Lin and P. J. Antsaklis. Stability and stabilizability of switched linear systems: A survey of recent results. IEEE Transactions on Automatic Control, 54(2):308-322, 2009.

[23] A. S. Morse. Supervisory control of families of linear set-point controllers - part 1: Exact matching. IEEE Trans. Automat. Contr, 41:1413-1431, 1998.

[24] K.S. Narendra, J. Balakrishnan, and M.K. Ciliz. Adaptation and learning using multiple models, switching, and tuning. IEEE Control Systems Magazine, 15:37 $-51,1995$.

[25] Yury Orlov, Yannick Aoustin, and Christine Chevallereau. Finite time stabilization of a perturbed double integrator - part i: Continuous sliding modebased output feedback synthesis. IEEE Trans. Automat. Contr., 56(3):614-618, 2011.

[26] S. Pettersson. Designing switched observers for switched systems using multiple lyapunov functions and dwell-time switching. In 2nd IFAC Conference on Analysis and Design of Hybrid Systems, 2006.

[27] A. Pisano and E. Usai. Sliding mode control: a survey with applications. Mathematics and Computers in Simulation, 81(5):954 - 979, 2011.

[28] Z. Sun and S.S. GE. Switched Linear Systems: Control and Design. SpringerVerlag, London, 2005.

[29] Zhendong Sun, Shuzhi Sam Ge, and Tong Heng Lee. Controllability and reachability criteria for switched linear systems. Automatica, 38(5):775-786, 2002.

[30] Aneel Tanwani, Hyungbo Shim, and Daniel Liberzon. Observability implies observer design for switched linear systems. In HSCC, pages 3-12, 2011.

[31] R. Vidal, A. Chiuso, S. Soatto, and S.Sastry. Observability of linear hybrid systems. In In Hybrid Systems: Computation and Control, LNCS, pages 526539. Springer Verlag, 2003.

[32] D. Vries, P.J.T. Verheijen, and A.J. den Dekker. Hybrid system modeling and identification of cell biology systems: perspectives and challenges. 15th IFAC Symposium on System Identification, pages 227 - 232, 2009.

[33] G. Xie and L. Wang. Necessary and sufficient conditions for controllability and observability of switched impulsive control systems. IEEE Transactions on Automatic Control, 49(6):960-966, 2004. 